\title{
A Complication of Paracentesis Due to Hyperfibrinolysis: Hemoperitoneum
}

\section{Elliot Smith ${ }^{1}$, Stephen H Caldwell ${ }^{2}$ and Neeral L Shah ${ }^{2 *}$}

${ }^{1}$ Department of Medicine, University of Virginia, Charlottesville, VA, USA

${ }^{2}$ Division of Gastroenterology and Hepatology, University of Virginia, Charlottesville, VA, USA

\begin{abstract}
Paracentesis is a commonly performed procedure associated with minimal risk of complication. We report a case of a cirrhotic patient who developed hemoperitoneum after paracentesis. This adverse outcome was attributed to a state of hypefibrinolysis. We describe a patient with end stage liver disease and ascites who developed a severe bleeding complication from a large volume paracentesis. Five days after the paracentesis, the patient developed a large abdominal wall hematoma. The patient was treated with a course of systemic epsilonaminocaproic acid and the bleeding resolved. Hyperfibrinolysis is the phenomenon of excessive clot breakdown and has been noted to increase the risk of procedural complications. The diagnosis of hyperfibrinolysis is clinically determined and therefore is often overlooked. This case demonstrates the need for increased recognition of this entity to ensure rapid and proper treatment of hyperfibrinolysis with anti-fibrinolytics.
\end{abstract}

Keywords: Paracentesis; Hemoperitoneum; Hyperfibrinolysis

\section{Introduction}

Paracentesis is a common and effective procedure used for diagnostic evaluation and therapeutic management of ascites. It is a relatively safe procedure that carries a minimal bleeding risk, though this risk is amplified in the setting of a hyperfibrinolytic state [1]. Hyperfibrinolysis is a phenomenon of excessive clot breakdown that is clinically manifested by mucocutaneous bleeding. The following case is that of a patient with cirrhosis who developed a large abdominal hematoma after a paracentesis in the setting of a hyperfibrinolytic state.

\section{Case Report}

A 57 year-old woman with a past history of alcoholic cirrhosis presented to the emergency department with a chief complaint of a bleeding tongue ulcer. The patient had first noticed the bleeding ulcer one week prior to presentation. In the interval, she reported decreased oral intake and the development of several bruises over her arms and legs, attributed to recent falls. Physical exam was notable for a large and actively oozing ulcer on the right side of the patient's tongue; diffuse ecchymosis of all four extremities; and a small, slowly bleeding laceration over her left foot.

Laboratory results on admission were significant for an immeasurable International Normalized Ratio (INR) of $>9.3$, prothrombin Time $>140$, and a fibrinogen level $<35 \mathrm{mg} / \mathrm{dL}$. She also had a hemoglobin level of $7.5 \mathrm{~g} / \mathrm{dl}$ and a platelet count of $28,000 / \mu \mathrm{L}$. She was subsequently transfused 2 units of packed red blood cells, 2 units of platelets, 2 units of cryoprecipitate, and two doses of IV vitamin K. Her laboratory values improved to an INR of 3.6, fibrinogen of 89, PTT of 50.9 , and platelets of 49 . Oral bleeding was controlled with topical epsilon-aminocaproic acid (EACA). In preparation for discharge, a therapeutic paracentesis was performed in the left lower quadrant via ultrasound guidance. A total of $3.5 \mathrm{~L}$ of fluid, bilious in colour, was successfully removed without immediate complication.

Five days after discharge the patient returned to the emergency department with a large abdominal wall hematoma (Figure 1). She reported that the bruising started at the site of paracentesis and gradually spread across her entire abdomen. Laboratory data was again notable for a low fibrinogen level of $54 \mathrm{mg} / \mathrm{dL}$ and elevated INR and PTT, 3.5 and 97.3 respectively. Hemoglobin had decreased to $6.7 \mathrm{~g} / \mathrm{dL}$, leading to transfusion of 2 units of packed red blood cells. A CT scan was performed and localized the hematoma to the subcutaneous tissue without involvement of the peritoneal cavity (Figure 2). The patient again received cryoprecipitate to replete her fibrinogen stores. She was treated with systemic epsilon-aminocaproic acid, with an oral loading dose of $4 \mathrm{mg}$ followed by $1 \mathrm{mg}$ every 6 hours for a 7-day total course. The size of the hematoma stabilized with these therapies and slowly resolved over the ensuing weeks (Figure 3). The patient presented again on multiple subsequent occasions with oral mucosal and cutaneous bleeding, which were treated with local EACA.

\section{Discussion}

The case above presents a cirrhotic patient who developed a large abdominal hematoma due to paracentesis in the setting of a bleeding diathesis. Hemostasis in cirrhosis is a complex pathway with alterations in both pro- and anti-hemostatic pathways leading to a new "rebalanced" state $[2,3]$. On initial presentation, coagulopathy was manifested by a combination of thrombocytopenia, elevated INR/PTT, and hyperfibrinolysis. Therapy focused on all three of these derangements, with transfusion of platelets, cryoprecipitate to replete fibrinogen, and addition of vitamin $\mathrm{K}$ to aid the production of coagulation factors. Paracentesis was performed after conventional measures of the coagulopathy and thrombocytopenia had improved, but the patient developed a large abdominal wall hematoma in spite of the treatment of these parameters. This complication was likely due to a hyperfibrinolytic state as evidenced by mucocutaneous bleeding and by the delayed development of bleeding after the procedure.

Fibrinolysis, the process of clot resolution, is altered in liver disease. The pathway for fibrinolysis is complex and involves interaction

*Corresponding author: Neeral L Shah, Division of Gastroenterology and Hepatology, University of Virginia, Charlottesville, VA, USA, Tel: 434-924-0316; Fax: 434-244-7546; E-mail: ns3zt@hscmail.mcc.virginia.edu

Received July 22, 2013; Accepted August 07, 2013; Published August 09, 2013

Citation: Smith E, Caldwell SH, Shah NL (2013) A Complication of Paracentesis Due to Hyperfibrinolysis: Hemoperitoneum. J Blood Disorders Transf S3: 005. doi:10.4172/2155-9864.S3-005

Copyright: $\odot 2013$ Smith E, et al. This is an open-access article distributed under the terms of the Creative Commons Attribution License, which permits unrestricted use, distribution, and reproduction in any medium, provided the original author and source are credited. 


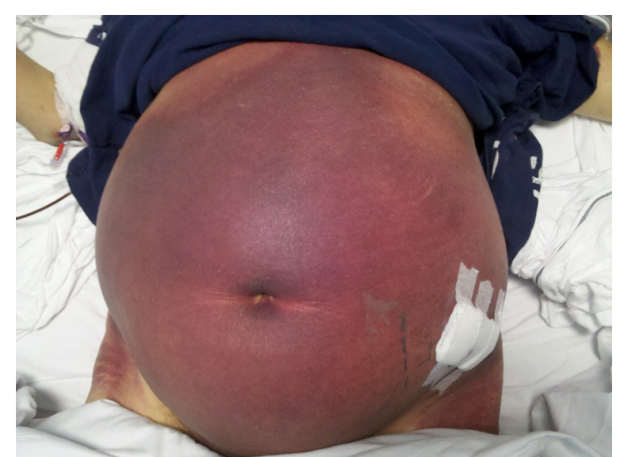

Figure 1: Large abdominal wall hematoma

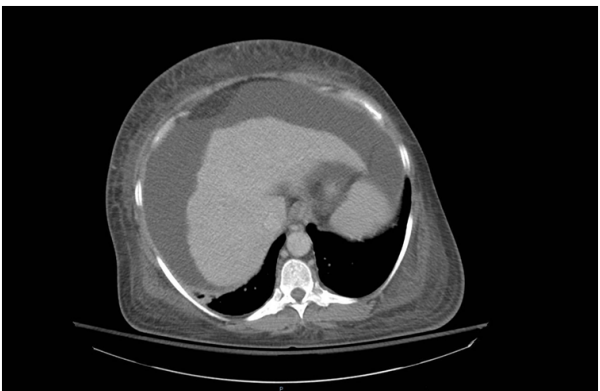

Figure 2: A CT scan was performed and localized the hematoma to the subcutaneous tissue without involvement of the peritoneal cavity

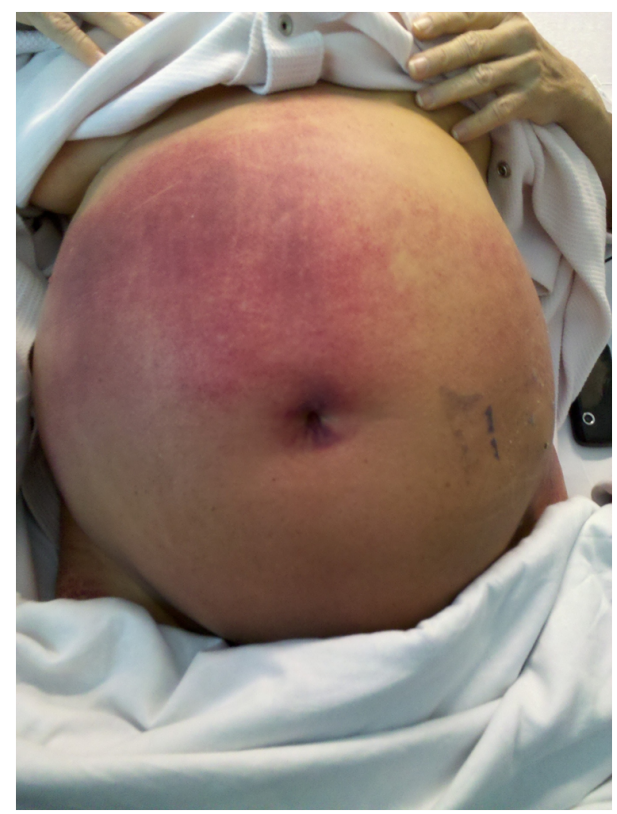

Figure 3: The size of the hematoma stabilized with these therapies and slowly resolved over the ensuing weeks

between both pro- and anti-fibrinolytic factors. Pro-fibrinolytic pathway alteration includes decreased production of factors produced in the liver, such as alpha-2 plasmin inhibitor and thrombin-activatable fibrinolysis inhibitor (TAFI). This is coupled with elevated levels of tissue plasminogen activator (tPA) that is presumably due to decreased hepatic clearance. Anti-fibrinolytic derangements include decreased liver synthesis of plasminogen and an increased release of PAI-1 as an acute phase response. In general, it is assumed that the balance of pro- and anti-fibrinolytic derangements in liver disease leads to a net pro-fibrinolytic state. However, the rate of true hyperfibrinolysis in cirrhosis is debated due to a lack of objective diagnostic methods. At present, no commercially available test exists to assess the fibrinolysis pathway in a "global" sense; rather, current modalities each evaluate particular components of the pathway [3-6]. Therefore, the diagnosis of hyperfibrinolysis is clinically based and should be considered in the setting of excessive mucosal bleeding, puncture wound bleeding, or delayed procedure-related bleeding $[7,8]$. Saliva and ascites have been noted to have inherent fibrinolytic properties, which may predispose patients to bleeding in the oral and abdominal cavities $[8,9]$. This phenomenon was demonstrated in the presented case as the patient developed both oral mucosal and abdominal bleeding that was a challenge to control.

Potential treatments of active fibrinoloysis include the lysine derivatives epsilon- aminocaproic acid (EACA) and tranexamic acid. Unfortunately, there are few clinical studies of these agents in liver disease, and the therapeutic dosing range has yet to be firmly established. Both of these drugs work by competitive inhibition of the conversion of plasminogen to plasmin $[4,8]$. EACA has been studied in a group of 37 patients with bleeding episodes attributed to hyperfibrinolysis, the majority of which were mucocutaneous in location [10]. Of these patients, 34 experienced resolution of bleeding with EACA treatment. The suggested dose from this study was $1 \mathrm{~g}$ every 6 hours, which was applied in the treatment of the patient described above. Aprotinin is another anti-fibrinolytic agent whose efficacy lies in direct inhibition of plasmin and kallirein; this agent was removed from the US market, however, after research concluded that the drug was associated with higher rates of mortality $[2,4]$.

In general, paracentesis is viewed as a relatively safe procedure with a bleeding risk less than $1 \%$ in the setting of cirrhosis $[1,11-13]$ AASLD guidelines do not recommend correcting coagulopathies prior to paracentesis, though special consideration is given to patients with evidence of hyperfibrinolysis; in such circumstances, treatment with EACA is recommended [12]

\section{Conclusion}

The case presented above illustrates the potential complication of bleeding due to a hyperfibrinolytic state in the setting of paracentesis, an otherwise low-risk procedure, ultimately warranting transfusion of blood products. Hyperfibrinolysis is associated with liver disease, although its frequency remains poorly defined as a result of suboptimal laboratory studies. This case emphasizes the need for the development of global measures of coagulation in liver disease and the importance of recognition and treatment of hyperfibrinolysis in procedures.

\section{References}

1. De Gottardi A, Thévenot T, Spahr L, Morard I, Bresson-Hadni S, et al. (2009) Risk of complications after abdominal paracentesis in cirrhotic patients: a prospective study. Clin Gastroenterol Hepatol 7: 906-909.

2. Bennani-Baiti N, Daw HA (2011) Primary hyperfibrinolysis in liver disease: a critical review. Clin Adv Hematol Oncol 9: 250-252.

3. Tripodi A, Mannucci PM (2011) The coagulopathy of chronic liver disease. N Engl J Med 365: 147-156.

4. Ferro D, Celestini A, Violi F (2009) Hyperfibrinolysis in liver disease. Clin Liver Dis 13: 21-31 
Citation: Smith E, Caldwell SH, Shah NL (2013) A Complication of Paracentesis Due to Hyperfibrinolysis: Hemoperitoneum. J Blood Disorders Transf S3: 005. doi:10.4172/2155-9864.S3-005

Page 3 of 3

5. Tripodi A (2009) Tests of coagulation in liver disease. Clin Liver Dis 13: 55-61.

6. Lisman T, Leebeek FW (2007) Hemostatic alterations in liver disease: a review on pathophysiology, clinical consequences, and treatment. Dig Surg 24: 250258

7. Caldwell SH, Hoffman M, Lisman T, Macik BG, Northup PG, et al. (2006) Coagulation disorders and hemostasis in liver disease: pathophysiology and critical assessment of current management. Hepatology 44: 1039-1046.

8. Shah NL, Caldwell SH, Berg CL (2009) The role of anti-fibrinolytics, rFVIla and other pro-coagulants: prophylactic versus rescue? Clin Liver Dis 13: 87-93.

9. Agarwal S, Joyner KA Jr, Swaim MW (2000) Ascites fluid as a possible origin for hyperfibrinolysis in advanced liver disease. Am J Gastroenterol 95: 3218-3224.
10. Gunawan B, Runyon B (2006) The efficacy and safety of epsilon-aminocaproic acid treatment in patients with cirrhosis and hyperfibrinolysis. Aliment Pharmacol Ther 23: 115-120.

11. Lin $\mathrm{CH}$, Shih FY, Ma MH, Chiang WC, Yang CW, et al. (2005) Should bleeding tendency deter abdominal paracentesis? Dig Liver Dis 37: 946-951.

12. Runyon BA; AASLD Practice Guidelines Committee (2009) Management of adult patients with ascites due to cirrhosis: an update. Hepatology 49: 20872107.

13. McGibbon A, Chen GI, Peltekian KM, van Zanten SV (2007) An evidencebased manual for abdominal paracentesis. Dig Dis Sci 52: 3307-3315.
Citation: Smith E, Caldwell SH, Shah NL (2013) A Complication of Paracentesis Due to Hyperfibrinolysis: Hemoperitoneum. J Blood Disorders Transf S3: 005. doi:10.4172/2155-9864.S3-005

This article was originally published in a special issue, Thrombolic \& Thrombocyłopenic Purpurea handled by Editor(s). Dr. Han-Mou Tsai, Pennsylvania State University, USA. 